\title{
A REMARK ON p-VALENT FUNCTIONS
}

\author{
JAMES A. JENKINS* and KÔTARO OIKAWA
}

(Received 8 August 1969)

Communicated by E. Strzelecki

In the theory of multivalent functions there are several different levels of postulates for $p$-valency. Perhaps the most well-known is the class of mean $p$ valent functions in the sense of Spencer [8] (we shall refer to them as areally mean $p$-valent functions), whose basic properties are found, e.g., in Hayman [4]. Recently Eke [1,2] extended to these functions a number of results which had been known for circumferentially mean $p$-valent functions.

On the other hand, Garabedian-Royden [3] and Jenkins [5] have introduced a wider class, for which they discussed the extension of Koebe's 1/4-theorem. Functions in this class are referred to as weakly mean $p$-valent functions by the former, and logarithmically areally mean $p$-valent functions by the latter. There are various other properties of areally mean $p$-valent functions which are satisfied by those functions also.

In the present paper, we shall discuss a negative aspect of logarithmically areally mean $p$-valent functions. It will be shown that the above mentioned result of Eke cannot be extended to those functions.

We shall also give a glance at $s$-dimensionally mean $p$-valent functions, discussed in Spencer [8], which lie in between areally mean $p$-valent functions and logarithmically areally mean $p$-valent functions.

\section{2}

Given a regular function $f$ on the unit disc $|z|<1$, let $n(w)$ be the number of $w$-points counted with multiplicity, and consider its circumferential mean

$$
p(R)=\frac{1}{2 \pi} \int_{0}^{2 \pi} n\left(R e^{i \theta}\right) d \theta,
$$

$0 \leqq R<\infty$. It is a non-negative lower-semicontinuous function and is such that $p(R)>0$ if and only if there exists $z$ satisfying $R=|f(z)|$.

* Research supported in part by the National Science Foundation. 
If there exists a number $p>0$ such that

$$
\int_{0}^{R} p(R) d\left(R^{2}\right) \leqq p \pi R^{2}
$$

for $R>0, f$ is called an areally mean p-valent function. It has at most $[p]$ zeros and satisfies the following basic inequality (see Hayman [4, p. 23]):

$$
\frac{1}{p}\left(\log \frac{R_{2}}{R_{1}}-\frac{1}{2}\right) \leqq \int_{R_{1}}^{R_{2}} \frac{d R}{R p(R)}
$$

for every $R_{1}, R_{2}$ with $0<R_{1}<R_{2}$.

If there exists $p>0$ such that

$$
\int_{R_{1}}^{R_{2}} \frac{p(R)}{R} d R \leqq p\left(\log \frac{R_{2}}{R_{1}}+\frac{1}{2}\right)
$$

for every $R_{1}$ and $R_{2}$ with $0<R_{1}<R_{2}$, we shall call flogarithmically areally mean p-valent. As appears implicitly in Hayman [4, p. 33], (A) implies (L). Further a function with $(L)$ has at most $[p]$ zeros and, as Schwarz's inequality

$$
\left(\log \frac{R_{2}}{R_{1}}\right)^{2} \leqq \int_{R_{1}}^{R_{2}} \frac{p(R)}{R} d R \cdot \int_{R_{1}}^{R_{2}} \frac{d R}{R p(R)}
$$

shows, satisfies (B). As a consequence, all the theorems in Chapter 2 of Hayman [4] are true for logarithmically areally mean $p$-valent functions.

If $p$ is a positive integer, a function $f$ with expansion

$$
f(z)=z^{p}+a_{p+1} z^{p+1}+\cdots
$$

about the origin will be referred to as normalized.

Clearly for a function with (N) and (A) there exists an $R_{0}>0$ such that $p(R)=p$ for $R$ with $0 \leqq R \leqq R_{0}$ and

$$
\frac{1}{p} \log \frac{R}{R_{0}} \leqq \int_{R_{0}}^{R} \frac{d R}{R p(R)}
$$

for every $R \geqq R_{0}$.

We shall call a function normalized logarithmically areally mean p-valent if it satisfies (N) and, for some $R_{0}$,

$$
\left\{\begin{array}{l}
p(R)=p \text { for every } R \text { with } 0 \leqq R \leqq R_{0} \\
\int_{R_{0}}^{R} \frac{p(R) d R}{R} \leqq p \log \frac{R}{R_{0}}
\end{array}\right.
$$


for every $R \geqq R_{0}$. It is to be noted that neither of the implications $(\mathrm{N}, \mathrm{L}) \leftrightarrows$ $\left(\mathrm{N}, \mathrm{L}^{*}\right)$ holds $\left(\right.$ see $9^{\circ}$ ).

As before a function with $(\mathrm{N}, \mathrm{A})$ satisfies $\left(\mathrm{L}^{*}\right)$. A function with $\left(\mathrm{N}, \mathrm{L}^{*}\right)$ has a zero only at the origin and satısfies $\left(\mathrm{B}^{*}\right)$. Therefore the theorems in Chapter 2 of Hayman [4] continue to hold for normalized logarithmically areally mean $p$-valent functions also.

Under the assumption $(N)$, the condition $\left(L^{*}\right)$ is readily seen to be equivalent to

$$
\int_{0}^{R} \frac{p(R)-p}{R} d R \leqq 0 \quad \text { for every } R,
$$

which is the definition adopted by Garabedian-Royden [3].

We shall say that a $p$-valent function $f$ attains maximum growth in the direction $\varphi$ if

$$
\varlimsup_{r \rightarrow 1}(1-r)^{2 p}\left|f\left(r e^{i \varphi}\right)\right|>0 .
$$

For circumferentially mean $p$-valent functions (i.e., $p(R) \leqq p$ for every $R>0$ ) Hayman [4], and for areally $p$-valent functions Eke [1, 2] recently, proved that (1) implies regularity of growth, namely the existence of the finite non-zero limit

$$
\lim _{r \rightarrow 1}(1-r)^{2 p}\left|f\left(r e^{i \varphi}\right)\right|
$$

We shall show that this conclusion does not hold for a function with (L) or $\left(\mathrm{N}, \mathrm{L}^{*}\right)$ :

THEOREM 1. There exists a logarithmically areally mean p-valent function as well as a normalized logarithmically areally mean p-valent function which attain maximum growth in direction $\varphi$ yet do not have the limit (2).

\section{5}

To prove Theorem 1 by constructing counter-examples we need some preparation, which begins with the quotation of a result of Eke [1, Theorem 3] as follows:

If a regular function on $|z|<1$ has only a finite number of zeros and satisfies

$$
\varlimsup_{r \rightarrow 1}\left|f\left(r e^{i \varphi}\right)\right|=\infty, \int_{R_{0}}^{\infty} \frac{d R}{R p(R)}=\infty
$$

for some $R_{0}$ with $p\left(R_{0}\right)>0$, then the limit

$$
\alpha=\lim _{r \rightarrow 1}\left(\int_{R_{0}}^{\left|f\left(r e^{i \varphi}\right)\right|} \frac{d R}{R p(R)}-2 \log \frac{1}{1-r}\right)
$$

exists including the possibility of $\alpha=-\infty$. 
Notice that, if (3) holds for some $R_{0}$, then it does also for every $R_{0}$ with $p\left(R_{0}\right)>0$. The value of $\alpha$ depends on $R_{0}$, but whether or not $\alpha>-\infty$ is independent of $R_{0}$.

This result indicates that if the growth is measured by means of the integral in (4), then the growth is regular whenever $\alpha>-\infty$, and the case $\alpha>-\infty$ corresponds to the case where $f$ attains maximum growth so measured in direction $\varphi$.

We now compare these with (2) and (1).

LEMMA 1. For a function $f$ satisfying $(B)$, a necessary and sufficient condition for (1) is the validity of

$$
\varlimsup_{r \rightarrow 1}\left(\int_{R_{0}}^{\left|f\left(r e^{i \varphi}\right)\right|} \frac{d R}{R p(R)}-2 \log \frac{1}{1-r}\right)>-\infty
$$

and

$$
\varlimsup_{R \rightarrow \infty}\left(\frac{1}{p} \log R-\int_{R_{0}}^{R} \frac{d R}{R p(R)}\right)>-\infty
$$

for every, of equivalently some, $R_{0}$ with $p\left(R_{0}\right)>0$.

For a function f satisfying $\left(B^{*}\right)$, the same is true with respect to the $R_{0}$ involved in $\left(B^{*}\right)$.

Proof. It is immediate if we compare (4) with

$$
\varlimsup_{r \rightarrow 1}\left(\frac{1}{p} \log \left|f\left(r e^{i \varphi}\right)\right|-2 \log \frac{1}{1-r}\right)>-\infty,
$$

which is equivalent to (1). Notice that either $\left(1^{\prime}\right)$ or (5), (6) implies (3), so that the limit of (5) always exists.

LEMMA 2. For a function $f$ satisfying $(1)$ and $(L)$, the existence of the finite non-zero limit (2) is equivalent to the existence of the finite limit

$$
\lim _{R \rightarrow \infty}\left(\int_{R_{0}}^{R} \frac{p(R)}{R} d R-p \log R\right)
$$

for every, or equivalently some, $R_{0}$ with $p\left(R_{0}\right)>0$.

For a function $f$ satisfying $(1)$ and $\left(L^{*}\right)$, the same is true with respect to the $R_{0}$ involved in $\left(L^{*}\right)$.

Proof. On comparing (4) with

$$
\lim _{r \rightarrow 1}\left(\frac{1}{p} \log \left|f\left(r e^{i \varphi}\right)\right|-2 \log \frac{1}{1-r}\right)>-\infty,
$$


which is equivalent to the existence of the finite non-zero limit (2), we conclude as before that $\left(2^{\prime}\right)$ is equivalent to the existence of the finite limit

$$
\lim _{R \rightarrow \infty}\left(\frac{1}{p} \log R-\int_{R_{0}}^{R} \frac{d R}{R p(R)}\right) .
$$

On the other hand, the right-hand side of

$$
\begin{aligned}
& \int_{R_{0}}^{R} \frac{(p(R)-p)^{2}}{R p(R)} d R \\
& \quad=\left(\int_{R_{0}}^{R} \frac{p(R)}{R} d R-p \log \frac{R}{R_{0}}\right)+p^{2}\left(\int_{R_{0}}^{R} \frac{d R}{R p(R)}-\frac{1}{p} \log \frac{R}{R_{0}}\right)
\end{aligned}
$$

is bounded since (6) and either (L) or $\left(\mathrm{L}^{*}\right)$ are satisfied. Since the integrand of the left-hand side is non-negative, the limit for $R \rightarrow \infty$ of the integral exists. Accordingly the existence of (8) is equivalent to that of the first term of the right-hand side of (9). Q.E.D.

Actually Eke [2, Theorem 1] showed the existence of (7) for areally mean $p$-valent functions with (1), and proved that (1) implies (2).

In order to prove Theorem 1 , it suffices to construct a function $f$ which satisfies (5), (6), and either (L) or ( $\left.L^{*}\right)$, yet does not have the limit (7).

Except for (5), these conditions are geometric properties of the Riemann surface covering the $w$-plane. There is a case, even though very limited, where a sufficient condition for (5) (i.e., $\alpha>-\infty$ in (4)) also is obtained in geometric terms.

Suppose a regular function $f$ on $|z|<1$ has only a finite number of zeros. Take $r_{0}>0$ such that $f$ does not vanish on the annulus $r_{0}<|z|<1$. Let $D$ be the domain obtained from this annulus cut along the ray $\arg z=\varphi+\pi$. Clearly

$$
\zeta=\log f(z)
$$

is single valued and regular on $D$. We assume that this function is univalent. Furthermore we require the image domain to have the following shape: there exist positive lower-semicontinuous functions $\theta_{1}$ and $\theta_{2}$ on $-\infty<\xi<\infty$ having the property that the image domain is contained in $\Delta=\{\zeta=\xi+i \eta \mid-\infty<\xi<\infty$, $\left.-\theta_{1}(\xi)<\eta<\theta_{2}(\xi)\right\}$, contains $\left\{\zeta \in \Delta \mid \xi_{0} \leqq \xi\right\}$ for some $\xi_{0}$, and such that the point $z=e^{i \varphi}$ corresponds to $\zeta=+\infty$.

LEMMA 3. If a function $f$ with the above properties satisfies in addition the following conditions, then (5) holds: There exist $0<m$ and $M<\infty$ such that

$$
m<\theta_{k}(\xi)<M, \quad k=1,2
$$


for $\xi \geqq \xi_{0}$, and there exists $V<\infty$ such that the total variation $V_{k}\left(\xi_{1}, \xi_{2}\right)$ of $\theta_{k}$ over the interval $\left[\xi_{1}, \xi_{2}\right]$ satisfies

$$
V_{k}\left(\xi_{1}, \xi_{2}\right) \leqq V, \quad k=1,2
$$

for every $\xi_{1}, \xi_{2}$ with $\xi_{0} \leqq \xi_{1}<\xi_{2}$.

Proof. Map the unit disk cut along the radius $\arg z=\varphi+\pi$ by

$$
Z=\frac{1}{2} \log \frac{z e^{-i \varphi}}{\left(1-z e^{-i \varphi}\right)^{2}}
$$

onto the strip $S=\left\{Z|| \operatorname{Im} Z \mid<\frac{1}{2} \pi\right\}$. Apply the Second Fundamental Inequality of Ahlfors (see Jenkins-Oikawa [6]) to the conformal mapping $Z \rightarrow \zeta$. On setting $X^{\prime}(\xi)=\inf \{\operatorname{Re} Z \mid \operatorname{Re} \zeta(Z)=\xi\}, X^{\prime \prime}(\xi)=\sup \{\operatorname{Re} Z \mid \operatorname{Re} \zeta(Z)=\xi\}$, and $\Theta=$ $\theta_{1}+\theta_{2}$, we obtain

$$
\frac{X^{\prime \prime}(\xi)-X^{\prime}\left(\xi^{*}\right)}{\pi} \leqq \int_{\xi^{*}}^{\xi} \frac{d \xi}{\Theta(\xi)}+\frac{V M}{m^{2}}+\frac{4 M}{m}
$$

for every $\xi$ and $\xi^{*}$ such that $\xi_{0}+2 M<\xi^{*}<\xi$. Observe that $\Theta(\xi)=2 \pi p(R)$ if $\xi=\log R$ and $\frac{1}{2} \log \left(r(1-r)^{-2}\right) \leqq X^{\prime \prime}(\xi)$ if $R=\left|f\left(r e^{i \varphi}\right)\right|$. On taking $R_{0}$ with $\xi_{0}<\log R_{0}$ and then $\xi^{*}$ with $\log R_{0}<\xi^{*}$, we obtain

$$
2 \log \frac{r^{\frac{1}{2}}}{1-r} \leqq \int_{R_{0}}^{\left|f\left(r e^{i \Phi}\right)\right|} \frac{d R}{R p(R)}+\text { const, }
$$

which implies (5).

Now we are in a position to prove Theorem 1 . Consider a positive lower-semicontinuous function $\Theta(\xi)$ on $-\infty<\xi<\infty$ such that $\Theta(\xi)=2 \pi p=$ const. for $\xi<0$. Let $g$ be a conformal mapping of $|z|<1$ onto the domain $\Delta=\{\zeta=$ $\xi+i \eta|-\infty<\xi<\infty,| \eta \mid<\Theta(\xi) / 2\}$ such that $z=e^{i \varphi}$ corresponds to $\zeta=+\infty$. Set

$$
f=\exp g .
$$

Next, let $g^{*}$ be a conformal mapping of $|z|<1$ cut along the radius $\arg z=\varphi+\pi$ onto $\Delta$ such that $z=0, e^{i \varphi}$ correspond to $\zeta=-\infty,+\infty$, respectively, and that the radius $\arg z=\varphi$ corresponds to the real-axis in the $\zeta$-plane. If $p$ is a positive integer, the symmetry of $g^{*}$ guarantees the regularity of $\exp g^{*}$ on $|z|<1$, which then has a zero of multiplicity $p$ at the origin. On taking a constant $c_{0}$ suitably we can make

satisfy the condition $(\mathrm{N})$.

$$
f^{*}=\exp \left(g^{*}+c_{0}\right)
$$


For both these functions $f$ and $f^{*}$, we have

$$
2 \pi p(R)=\Theta(\xi) \quad \text { if } \xi=\log R .
$$

Accordingly the conditions (L), (L*), (6), (10), and (11) with respect to $R_{0}=1$ for $f$ and $R_{0}=\exp c_{0}$ for $f^{*}$ are respectively expressed as follows:

(15) The total variation of $\Theta(\xi)$ on any closed subinterval is bounded by a constant $V$.

The non-existence of the limit (7) is equivalent to

$$
\text { Non-existence of } \lim _{b \rightarrow \infty} \int_{0}^{b}(\Theta(\xi)-2 \pi p) d \xi \text {. }
$$

An example of a function $\Theta(\xi)$ with these properties is obtained by considering a step function as follows: Prepare sequences $\left\{\xi_{v}\right\}$ and $\left\{\varepsilon_{v}\right\}$ with $0=\xi_{0}<\xi_{1}<$ $\cdots \rightarrow \infty$ and $0<\varepsilon_{v}<1$, and set $\Theta(\xi)=2 \pi p$ if $\xi<0, \Theta(\xi)=2 \pi p\left(1+(-1)^{v} \varepsilon_{v}\right)$ if $\xi_{v-1}<\xi<\xi_{v}, v=1,2, \cdots$, and $\Theta\left(\xi_{v}\right)$ suitably so that the resulting function $\Theta(\xi)$ on $-\infty<\xi<\infty$ is positive and lower-semicontinuous. If the sequences satisfy, e.g.,

$$
\sum_{v=1}^{\infty} \varepsilon_{v}<\infty, \varepsilon_{v}\left(\xi_{v}-\xi_{v-1}\right)=\frac{1}{6}, \quad v=1,2, \cdots,
$$

then it is not difficult to see that $\Theta(\xi)$ satisfies $(12),\left(12^{*}\right),(13)-(16)$. The proof of Theorem 1 is herewith complete.

\section{9}

Incidentally, for a positive integer $p$, we can construct a $\Theta(\xi)$ with (12) but not $\left(12^{*}\right)$, and also one with $\left(12^{*}\right)$ but not $(12)$. Thus neither of $(\mathrm{N}, \mathrm{L})$ and $\left(\mathrm{N}, \mathrm{L}^{*}\right)$ implies the other.

Spencer's paper [9] contains a suggestion for another possible extension of areal mean $p$-valency. Let us call a regular function $f$ on the unit disc $|z|<1$ 
$s$-dimensionally mean p-valent $(s>0)$ if

$\left(\mathbf{A}_{\mathrm{s}}\right)$

$$
\int_{0}^{R} p(R) d\left(R^{s}\right) \leqq p \pi R^{s}
$$

for $R>0$.

Spencer [9] showed $\left(\mathrm{A}_{\mathrm{s}}\right) \Rightarrow\left(\mathrm{A}_{s^{\prime}}\right)$ if $0<s^{\prime} \leqq s$. On the other hand, by an argument similar to Hayman $\left[4\right.$, p. 33] we see that $\left(A_{s}\right)$ implies

$$
\int_{R_{1}}^{R_{2}} \frac{p(R)}{R} d R \leqq p\left(\log \frac{R_{2}}{R_{1}}+\frac{1}{s}\right)
$$

for every $R_{1}, R_{2}$ with $0<R_{1}<R_{2}$. On disregarding the last constant of $\left(\mathrm{L}_{\mathrm{s}}\right)$, we may say that $s$-dimensionally mean $p$-valent functions with $0<s<2$ are more general than areally mean $p$-valent functions, and essentially less general then logarithmically areally mean $p$-valent functions.

Observe that $\left(\mathrm{L}_{\mathrm{s}}\right)$ implies as before

$$
\frac{1}{p}\left(\log \frac{R_{2}}{R_{1}}-\frac{1}{s}\right) \leqq \int_{R_{1}}^{R_{2}} \frac{d R}{R p(R)},
$$

so that the theorems in Chapter 2 of Hayman [4] are true for these functions.

For a function with $\left(A_{s}\right)$ the reasoning of Eke [2, Theorem 1] is applicable mutatis mutandis to prove

THEOREM 2. For an $s$-dimensionally mean p-valent function $(0<s \leqq 2)$ which attains maximum growth in direction $\varphi$, the finite non-zero limit (2) exists.

REMARK. W. K. Hayman has informed the authors that closely related results have been obtained independently by V. R. Eke.

\section{References}

[1] B. G. Eke, 'Remarks on Ahlfors' distortion theorem', J. Anal. Math. 19 (1967), 97-134.

[2] B. G. Eke, 'The asymptotic behaviour of areally mean valent functions', J. Anal. Math. 20 (1967), 147-212.

[3] P. R. Garabedian and H. L. Royden, 'The one-quarter theorem for mean univalent functions', Ann. of Math. 59 (1954), 316-324.

[4] W. K. Hayman, Multivalent functions (Cambridge Univ. Press, 1958).

[5] J. A. Jenkins, 'On a conjecture of Spencer', Ann. of Math. 63 (1957), 405-410.

[6] J. A. Jenkins and K. Oikawa, 'On results of Ahlfors and Hayman', to appear in Illinois J. Math.

[7] J. A. Jenkins, 'On the growth of slowly increasing unbounded harmonic functions', Acta Math. 124 (1970), 37-63.

[8] D. C. Spencer, 'On finitely mean valent functions', Proc. London Math. Soc. 47 (1941), $201-211$.

[9] D. C. Spencer, 'On finitely mean valent functions', II, Trans. Amer. Math. Soc. 48 (1940), 418-435.

Washington University, St. Louis and University of Tokyo 